\title{
Effect of Surface Roughness on Electrochemical Adsorption/Desorption of Dopamine by Carbonaceous Electrodes
}

\author{
Alexander G. Zestos ${ }^{1 *}$, and Hanning Chen ${ }^{1 *}$ \\ ${ }^{1}$ Department of Chemistry, American University, Washington, DC 20016, USA \\ *Authors to whom correspondence should be addressed: (A. G. Zestos) zestos@american.edu \\ and (H. Chen) hchen@american.edu
}

\begin{abstract}
Electrochemical adsorption/desorption of dopamine by carbonaceous electrodes upon voltage variation is the key process of neurotransmitter detection through fast scan cyclic voltammetry. In the present study, ab initio molecular dynamics simulation empowered by imagecharge method was applied to calculate the adsorption/desorption free energy profile of dopamine and dopamine o-quinone at fixed electrode potentials using our newly developed open-source $\mathrm{CP} 2 \mathrm{~K}$ simulation package. It was found that the activation barriers for both adsorption and desorption were substantially reduced with increasing surface roughness of the carbonaceous electrodes. For example, on the flat graphene electrode, the activation barrier for dopamine adsorption at $\mathrm{V}_{0}=-0.4 \mathrm{~V}$ is $1.34 \mathrm{kcal} / \mathrm{mol}$, while its counterpart on the curved nanotube electrode drops to $0.82 \mathrm{kcal} / \mathrm{mol}$. Moreover, the diffusion coefficient of dopamine decreases by approximately $60 \%$ when it is moving close to the graphene electrode, while its diffusion is accelerated by up to $100 \%$ when the nanotube electrode is adopted. The faster diffusion alongside the reduced activation barrier greatly facilitates the electrochemically driven adsorption/desorption of dopamine by nanotube electrodes, in consistent with experimental findings that a rougher carbonaceous surface is critical for fast scan cyclic voltammetry.
\end{abstract}

Keywords: fast scan cyclic voltammetry, ab initio molecular dynamics, neurotransmitter detection 


\section{Introduction}

Dopamine is a neurotransmitter that has been postulated to be important for a wide variety of functions. Parkinson's disease, for example, is a movement disorder that arises from the death of dopaminergic neurons. ${ }^{1}$ Dopamine has also been found to increase in many behavioral and pharmacological states such as $\operatorname{sex}^{2}$, reward $^{3}$, reinforcement ${ }^{4}$, motivation ${ }^{5}$, fear ${ }^{6}$, and drug abuse (amphetamine and cocaine abuse) $)^{7}$ among many others ${ }^{8}$. Other neurochemicals such as serotonin play important roles for depression, while adenosine is thought to be cardioprotective ${ }^{9}$ and neuroprotective against heart attack and stroke ${ }^{10}$, respectively.

The detection of dopamine has been paramount to understanding complex behavioral and pharmacological states for many scientists. Techniques such as microdialysis ${ }^{11}$ coupled with high performance liquid chromatography (HPLC) $^{12}$ and liquid chromatography-tandem mass spectrometry (LC-MS/MS) ${ }^{13}$ allow for the sensitive and selective detection of dopamine and other neurotransmitters. However, these techniques cause great tissue damage and immune responses through the relatively large microdialysis probe. ${ }^{14}$ Positron emission tomography (PET) imaging has also been utilized for invasive dopamine measurements and D2-receptor binding, but this technique is relatively expensive and not available in basic laboratory settings. ${ }^{15}$

Carbon-fiber microelectrodes (CFMEs) have been the standard for neurotransmitter detection for over thirty years with fast scan cyclic voltammetry. Typically, a T-650 carbon fiber is aspirated into a glass capillary tube that is pulled to a fine taper, cut, and then epoxied before it is used as an electrode material. The carbon fiber is made from polyacrylonitrile polymer that is spun into fiber and pyrolyzed into conductive carbon fiber at temperatures over 1,000 K. CFMEs have been used for the detection of neurotransmitters in vivo, but they suffer from certain drawbacks. First and foremost, the carbon fiber contains more basal plane carbon that suffers from 
biofouling. During fouling, macromolecules such as proteins and biomolecules polymerize, thus coating the carbon fiber surface in a non-conductive polymer and hence, blocks adsorption of other biomolecules.

On the other hand, carbon nanotube fiber (CNTFMEs) and carbon nanotube yarn microelectrodes (CNTYMEs) have proven to enhance neurochemical detection in several ways with respect to CFMEs. Dipcoating functionalized CNTs suspended in Dimethylformamide (DMF) onto the surface of CFMEs has increased neurochemical detection and lowered the limit of detection of several neurochemicals. ${ }^{16}$ However, this process has created noisy measurements due to the heterogeneous CNT surface that was made up of many different sized carbon nanotubes. The development and usage of CNT yarn ${ }^{17}$ and fiber ${ }^{18}$ microelectrodes thoroughly enhanced neurotransmitter detection by providing a homogeneous carbon nanotube surface as an electrode material. The yarn was purchased commercially after being made from a furnace, while the fiber was made through a process called wet spinning with the use of a polymer, polyethyleneimine (PEI).

Both the PEI-CNTFMEs and the CNTYMEs displayed as sensitivity towards dopamine that was independent of the wave application frequency. This allows one to hypothesize that this effect is a function of the CNT electrode materials and not the method in which the electrode was prepared. Therefore, we hypothesize that the mechanism of adsorption to the surface of the CFMEs is different than on the surface of the CNTFME and CNTYMEs. The dopamine waveform (scanning at $400 \mathrm{~V} / \mathrm{sec}$ from $-0.4 \mathrm{~V}$ to $1.3 . \mathrm{V}$ at a wave application frequency of $10 \mathrm{~Hz}$ ) is optimal for dopamine adsorption since the negative holding potential allows for the preconcentration of dopamine to the surface of the CFME since the positively charged amino group of the catecholamine dopamine is protonated at a physiological $\mathrm{pH}$ of 7.4. Since the sensitivity towards 
dopamine or serotonin ${ }^{19}$ of CNTMEs are not affected by the wave application frequency, then it is postulated that dopamine may be adsorbing to their surface via a different mechanism. It is postulated that the temporal resolution of CFMEs is limited by their surface-induced retardation of the dopamine diffusion, while the temporal resolution of CNTFMEs is greatly improved due to their weaker aromatic ring $\pi-\pi$ stacking with dopamine. Furthermore, increased surface roughness on the surface of CNTMEs could be helping trap dopamine onto the surface of the electrode material, thus blocking desorption from the surface of the electrode material. Further experiments such as experimental testing and molecular dynamics simulations need to be performed in order to determine this mechanism to construct future electrode materials.

\section{Image-Charge QM/MM Method}

As the first step toward the computer-aided design of neurotransmitter detectors based on graphitic carbon electrodes, we have investigated the electrochemically driven adsorption/desorption of protonated dopamine onto two model carbonaceous surfaces, namely graphene and single-walled nanotube. In our computational study, the induced image-charge (IC) method $^{20}$ was employed in conjunction with the hybrid quantum mechanics/molecular mechanics $(\mathrm{QM} / \mathrm{MM})$ approach $^{21}$ to accurately model the mutual electronic polarization between the molecular adsorbates and electrode surfaces under arbitrary working potentials, $V_{0}$. Specifically, as illustrated in Fig. 3, a protonated dopamine molecule was modeled by density functional theory ${ }^{22}$ with PBE0 exchange-correlation functional ${ }^{23}$ and polarized double- $\zeta$ basis set, ${ }^{24}$ whereas a five-layer graphene electrode was described by discrete atom-centered image charges ${ }^{25}$ induced by the adsorbate's electrostatic potential. For numerical efficiency, each image charge, $\mathrm{q}_{\mathrm{i}}$, is smeared by a Gaussian distribution, yielding an MM electron density of 
$\rho_{M M}(r)=q_{i}(\alpha / \pi)^{\frac{3}{2}} e^{-\alpha\left|r-r_{i}\right|^{2}}$, where $\alpha$ was carefully chosen as 3.0 angstrom $^{-2}$ to afford numerical accuracy by suppressing charge overlap. By contrast, the QM electron density, $\rho_{Q M}(r)=\sum_{j}\left|\psi_{j}(r)\right|^{2}$, must be continuous due to the spatial continuity requirement of electron orbitals, $\psi_{j}(r)$. If the electrode is considered as a perfect conductor, no electrostatic potential gradient should be present inside the MM subdomain:

$$
V_{M M}(r)=\int \frac{\rho_{M M}\left(r^{\prime}\right)+\rho_{Q M}\left(r^{\prime}\right)}{\left|r-r^{\prime}\right|} d r^{\prime} \equiv V_{0}
$$

Therefore, the whole system's electrostatic energy, $E^{\text {elc. }}$

$$
E^{\text {elec }}=\frac{1}{2} \iint \frac{\left(\rho_{M M}(r)+\rho_{Q M}(r)\right)\left(\rho_{M M}\left(r^{\prime}\right)+\rho_{Q M}\left(r^{\prime}\right)\right)}{\left|r-r^{\prime}\right|} d r d r^{\prime}-\int V_{0} \rho_{M M}(r) d r
$$

can be minimized by varying the MM subsystem's image charges, $q_{i}$, which in turn polarize the QM subsystem. For instance, when $\mathrm{V}_{0}$ of the graphene electrode increases from $-0.4 \mathrm{~V}$ to $+1.3 \mathrm{~V}$ (Fig. 1), the electron density of its adsorbed dopamine molecule exhibits a significant drift towards the surface in concert with a notable change of the accumulated image charges from -2.18 to +6.17 . In addition, the same trend was also observed in the dopamine/nanotube adduct (Fig. 2), another promising candidate for neurotransmitter detector. Unless otherwise specified, all preliminary computational studies were performed using $\mathrm{CP} 2 \mathrm{~K},{ }^{26}$ an open-source molecular simulation package that is freely available for academic research.

\section{Dopamine Binding Free Energy as a Function of Electrode Surface Roughness}


Since the adsorption/desorption of protonated dopamine is electrochemically driven (Fig. 4), a critical factor to decide the responsiveness of its detector is the sensitivity of its binding affinity, $\Delta G_{b}$, to the electrode's working potential, $\mathrm{V}_{0}$. Although $\Delta G_{b}$ can be obtained, in theory, by sampling all possible atomistic configurations weighted by their energies, the complicated energy landscape of any realistic system makes the task computationally daunting. Umbrella sampling technique ${ }^{27}$ stands out as a reliable method for enhanced sampling, ${ }^{28}$ particularly for systems with well-defined reaction coordinates, $R_{C}$. For example, $R_{C}$ is naturally the distance between the center of mass (COM) of dopamine and the outer-layer graphene surface, $D_{M S}$, when a multi-layer graphene electrode is used. For a nanotube electrode, $R_{C}$ can be defined as the distance between dopamine's COM and the nanotube's axis. The basic idea of umbrella sampling $^{27}$ is to add a biased harmonic potential to a system's Hamiltonian in order to explore energetically unfavorable configurations that otherwise will be poorly sampled or even entirely unsampled: $H^{\prime}=H_{0}+k\left(R_{C}-R_{C}^{0}\right)^{2}$. After imposing the system at different positions along a pre-defined reaction coordinate, ${ }^{27}$ we can recover the free energy profile, $\Delta G_{b}\left(R_{C}\right)=-k_{B} T \ln P\left(R_{C}\right)$ through the weighted histogram analysis method, ${ }^{29}$ wherein the unbiased probability, $P\left(R_{C}\right)$, of finding the system at a given reaction coordinate is determined in a self-consistent manner. When protonated dopamine is probed by cyclic voltammetry, the electrode stays at its reference potential of $-0.4 \mathrm{~V}$ for a long time to attract and retain the adsorbate, before its potential is ramped up linearly to $+1.3 \mathrm{~V}$ for two purposes, namely the oxidation of protonated dopamine to protonated dopamine-o-quinone as well as the latter's desorption from the electrode. As shown in Fig. 3a, a flat electrode surface (Fig. 2a) at -0.4V attracts the protonated dopamine to an energy minimum at $\sim 4 \AA$ with $1.34 \mathrm{kcal} / \mathrm{mol}$ binding activation energy. After the electrode potential is increased to $+1.3 \mathrm{~V}$, the protonated dopamine is oxidized to protonated dopamine-o-quinone, which is then repelled to the bulk water phase with a desorption activation energy of $1.55 \mathrm{kcal} / \mathrm{mol}$. By contrast, for a carbon nanotube (Fig. 2d), the binding activation energy for protonated dopamine decreases to $0.82 \mathrm{kcal} / \mathrm{mol}$ while 
the desorption activation energy for protonated dopamine-o-quinone notably raises to $0.84 \mathrm{kcal} / \mathrm{mol}$ (Fig. 3b). The enhanced sensitivity of adsorption/desorption energy of dopamine/dopamine-o-quinone on carbon nanotube can be ascribed to its disruption of the water's hydrogen-bonding network, giving rise to a lower aqueous dielectric function for a poorer screening of electrode voltage. With this regard, a systematic examination of the surface effect on the adsorption/desorption profile is highly desired by varying the electrode's SR to 0.25 (Fig. 2b) and 0.75 (Fig. 2c). Moreover, the sensitivity of an electrode for dopamine detection can be roughly assessed by the conductivity, g, of the dopamine cation when the electrode potential, $\mathrm{V}$, is varied between $-0.4 \mathrm{~V}$ and $+1.3 \mathrm{~V}: g=J / V$, where $\mathrm{J}$ is the current density. In general, a higher conductivity is desired for a faster scan when the scan rate of cyclic voltammetry is limited by the adsorbate's adsorption/desorption. This assumption is usually valid for dopamine cation as its diffusion coefficient is at the scale of $10^{-6} \mathrm{~cm}^{2} / \mathrm{s},{ }^{30}$ which is readily outpaced by the interfacial electron transfer rate, $k_{e t}$, associated with its reduction/oxidation by the electrode. ${ }^{31}$ In fact, $k_{e t}$, can be estimated by our functional mode electron transfer (FMET) theory ${ }^{32}$ that has been successfully applied to the electron transfer between 6-methyl-azulene-2-carboxlic acid to $\mathrm{TiO}_{2}$ electrode. ${ }^{33}$

\section{Poisson-Nernst-Planck Electrodiffusion Theory for Cyclic Voltammetry}

According to the Poisson-Nernst-Planck (PNP) theory, ${ }^{34}$ the maximum conductivity, $g_{\max }$, of an ion at its steady non-equilibrium state is given by:

$$
g_{\max }=\frac{e^{2}}{k_{B} T L^{2}}\left\langle D\left(R_{C}\right)^{-1} e^{+\Delta G_{b}\left(R_{C}\right) / k_{B} T}\right\rangle^{-1}\left\langle e^{+\Delta G_{b}\left(R_{C}\right) / k_{B} T}\right\rangle^{-1}
$$

where $L$ is the diffusion length, $D\left(R_{C}\right)$ is the position-dependent diffusion coefficient along the diffusion pathway, and $\Delta G_{b}\left(R_{C}\right)$ is the associated free energy profile that can be obtained by the abovementioned umbrella sampling technique. Moreover, if the ion is treated as an overdamped Langevin oscillator under the imposed harmonic potential, its $D\left(R_{C}\right)$ can be estimated by the 
simplified Woolf-Roux equation: ${ }^{35} D\left(R_{C}\right)=\operatorname{var}\left(R_{C}\right) / \tau_{R_{C}}$, where $\operatorname{var}\left(R_{C}\right)$ is the variance of $R_{C}$, and $\tau_{R_{C}}$ is the exponentially fitted characteristic time of its autocorrelation function. Although the PNP theory has been successfully applied by PI Chen to investigate ion transport across a variety of biomolecular channels ${ }^{36-38}$ under static biased voltage, a correction term in the form of RandlesSevcik function, ${ }^{39,40} \zeta$, has to be added to Eq.3: $g=g_{\max } \zeta(D, S)$, to account for the effect of finite ion diffusion rate, $D$, when compared with the cyclic voltammetry scan rate, $S$. Numerically, $\zeta$ can be evaluated through an asymptotic expansion with controllable error bounds. ${ }^{41}$ As demonstrated by Fig. $4, \Delta G_{b}$ reaches its plateau value at around $R_{C}=10 \AA \approx \mathrm{L}$ for both graphene and nanotube electrodes, yielding an approximate diffusion characteristic time, $t_{D}=L^{2} / 4 D$, of $\sim 10^{-7} \mathrm{~s}$. Therefore, carbonaceous electrodes are well suited for ultra-fast dopamine detection with an upper scan rate limit of $\sim 10^{6} \mathrm{~V} \cdot \mathrm{s}^{-1}$ if one considers the typical electrode potential range of [$0.4 \mathrm{~V},+1.3 \mathrm{~V}]$. Interestingly, $D$ was found to decrease substantially for dopamine molecule when it moves from the bulk water phase to the surface of the graphene surface, suggesting a surfaceinduced retardation mechanism for the time-limiting step of dopamine detection on a smooth electrode surface. Our hypothesis gains immediate support from the notable difference on the profile of $D$ between graphene and nanotube, particularly at the near-surface domain as circled in green in Fig. 4. Apparently, dopamine enjoys a greater mobility on the three-dimensional carbon nanotube, whose surface is much rougher than that of the two-dimensional graphene sheet. Due to the remarkable $\pi-\pi$ aromatic ring stacking effect between dopamine and graphene, our calculated $D_{\text {surface }}$ is as small as $0.5 \times 10^{-6} \mathrm{~cm}^{2} / \mathrm{s}$ at the potential well of $\Delta \mathrm{G}_{\mathrm{b}}$. By contrast, $D_{\text {surface }}$ stays at $\sim 5.0 \times 10^{-6} \mathrm{~cm}^{2} / \mathrm{s}$ for nanotube that is in well line with $6.0 \times 10^{-6} \mathrm{~cm}^{2} / \mathrm{s},{ }^{42}$ the experimental diffusion coefficient of dopamine in pure water, $D_{\text {bulk }}$. The resemblance of $D_{\text {surface }}$ to $D_{b u l k}$ of dopamine in 
the presence of nanotube electrode might arise from the weaker $\pi-\pi$ aromatic ring stacking and disrupted hydrogen-bonding network. Therefore, from the molecular perspective, nanotube should be a much better candidate than graphene for dopamine detection, and this hypothesis has been supported by our experimental preliminary results. Moreover, the dynamic response of dopamine to a time-dependent electrode potential with arbitrary waveform will be modeled by our newly implemented dynamical local charge redistribution (DLCR) algorithm ${ }^{43}$ in $\mathrm{CP} 2 \mathrm{~K}$ software that has

enabled molecular dynamics simulation to approach the millisecond timescale, the period of a typical cyclic voltammetry waveform for dopamine detection. Similarly, our simulation protocol, once justified on dopamine adsorption/desorption, will be employed to other monoamine neurotransmitters such as norepinephrine, serotonin, and others.

\section{Conclusions:}

This works offers the first ever hybrid experimental/theoretical approach to investigate the adsorption of neurotransmitter onto microelectrodes. It will unveil a previously unknown mechanism of dopamine adsorption onto carbonaceous surfaces, thus facilitating the systematic design of ultra-fast detectors for other neurotransmitters.

\section{Acknowledgements}

This work was supported by the American University through start-up funds.

\section{Declaration of Interests:}

The authors declare no competing interests. 
Figures:

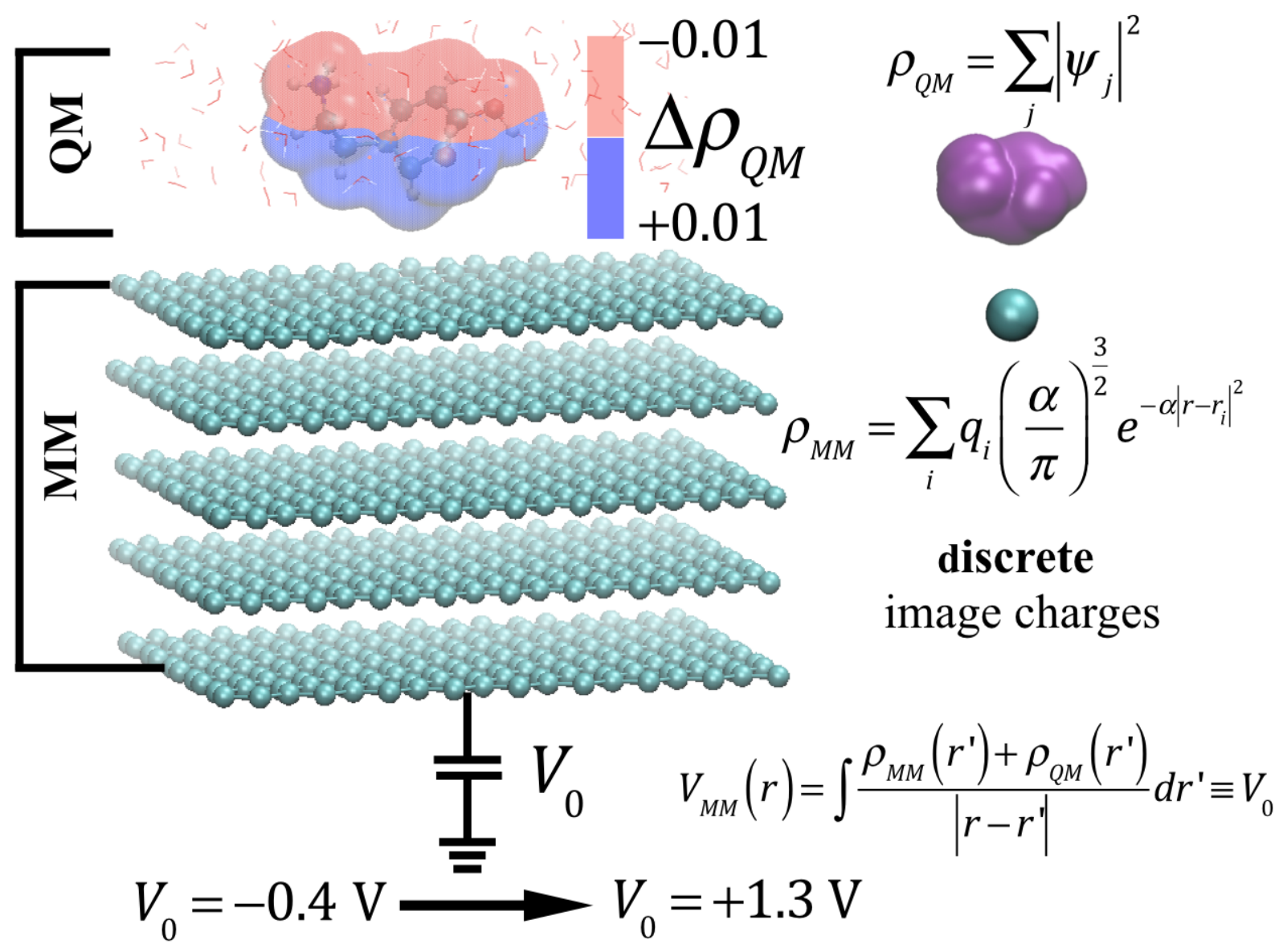

Figure 1. Simulated electron density change, $\Delta \boldsymbol{\rho}_{Q M}$, of a protonated dopamine molecule adsorbed onto a five-layer graphene electrode, when its working potential, $V_{0}$, increases from $-0.4 \mathrm{~V}$ to $+1.3 \mathrm{~V}$. 
(A)

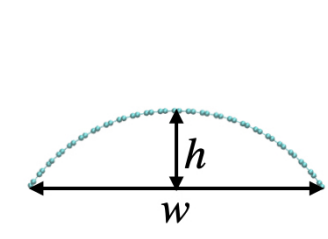

Surface Roughness:

$S R=\frac{2 h}{w}$
(B)

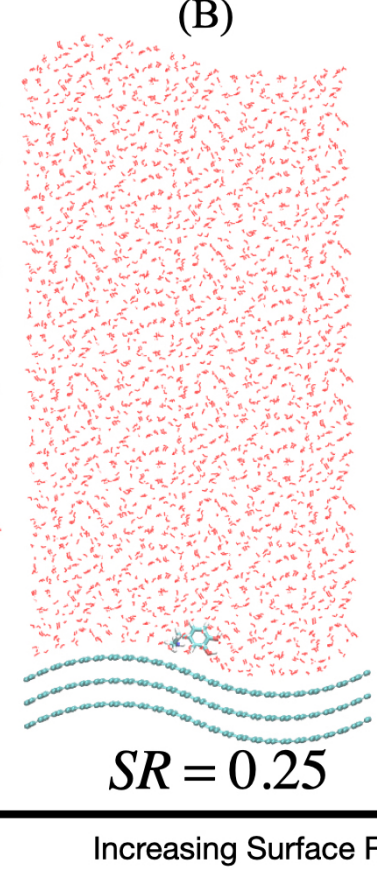

(C)

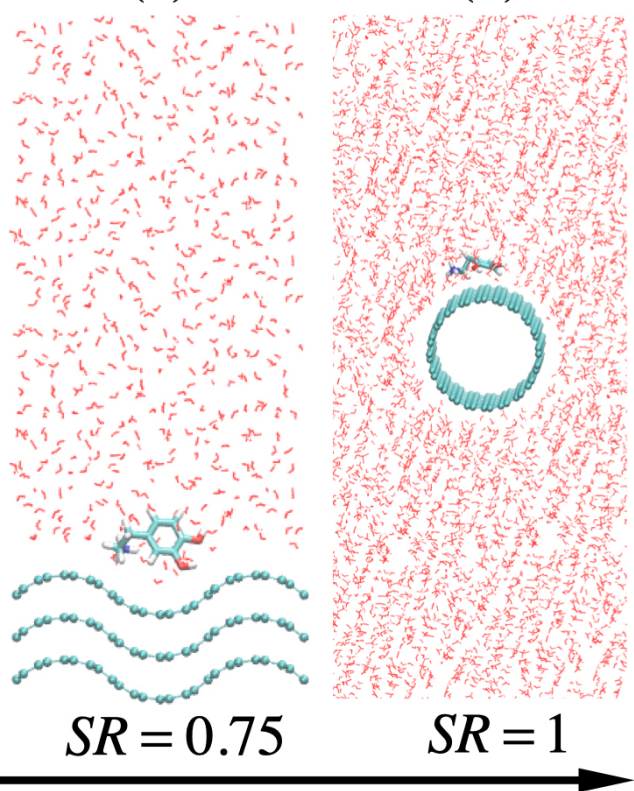

$S R=0.75$

(D)

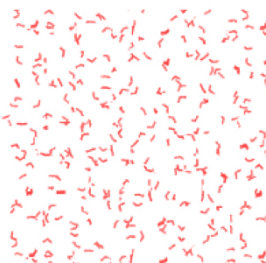

(1)

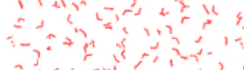

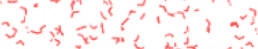
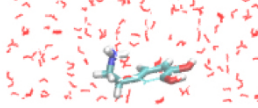

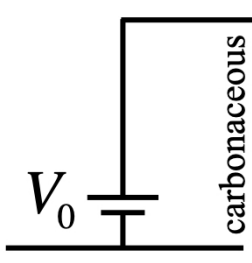

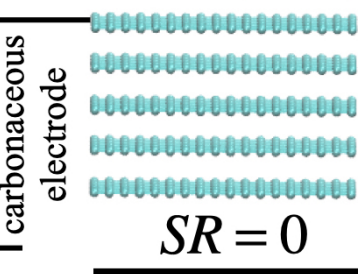

Figure 2. Four fully solvated electrode/neurotransmitter systems with various surface roughness (SR) quantified by the ratio between the height, $h$, and width, $w$, of the curved electrode surface. 


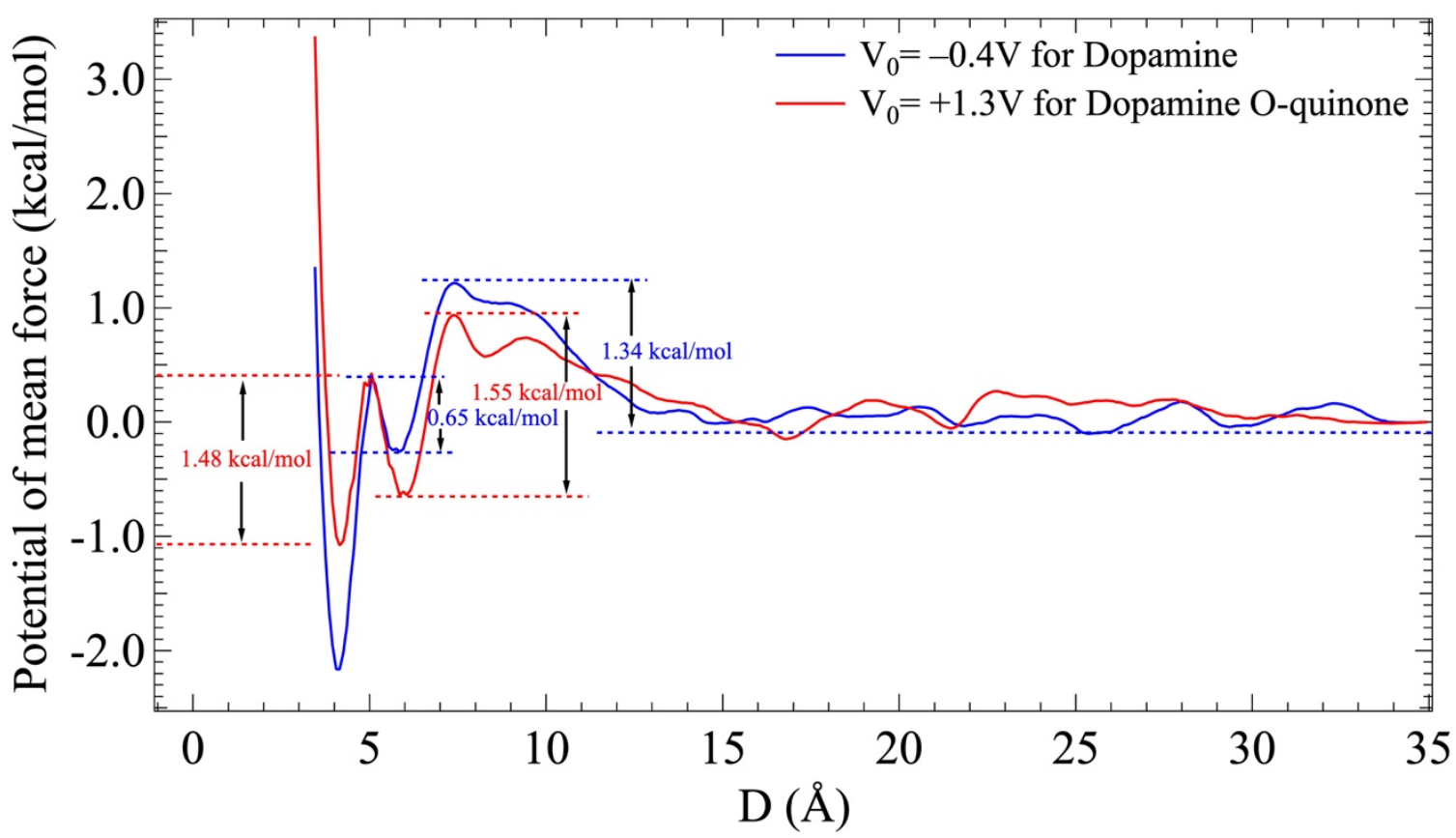

Figure 3(a). Calculated potential of mean force for dopamine at $\mathrm{V}_{0}=-0.4 \mathrm{~V}$, and for dopamine 0 quinone at $\mathrm{V}_{0}=+1.3 \mathrm{~V}$, both on a graphene electrode (Fig. 2a).

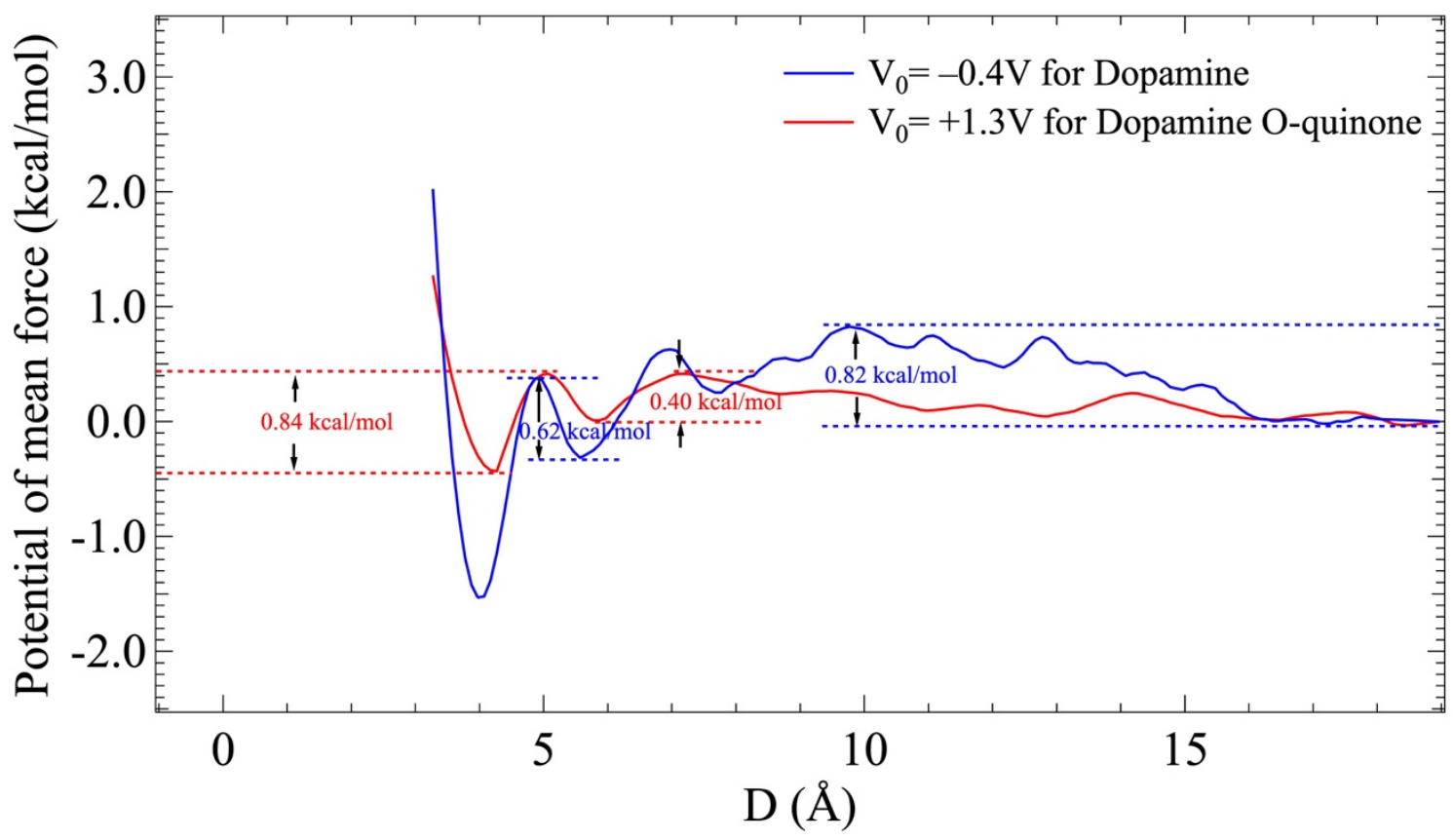

Figure 3(b). Calculated potential of mean force for dopamine at $\mathrm{V}_{0}=-0.4 \mathrm{~V}$, and for dopamine $0-$ quinone at $\mathrm{V}_{0}=+1.3 \mathrm{~V}$, both on a nanotube electrode (Fig. $2 \mathrm{~d}$ ). 

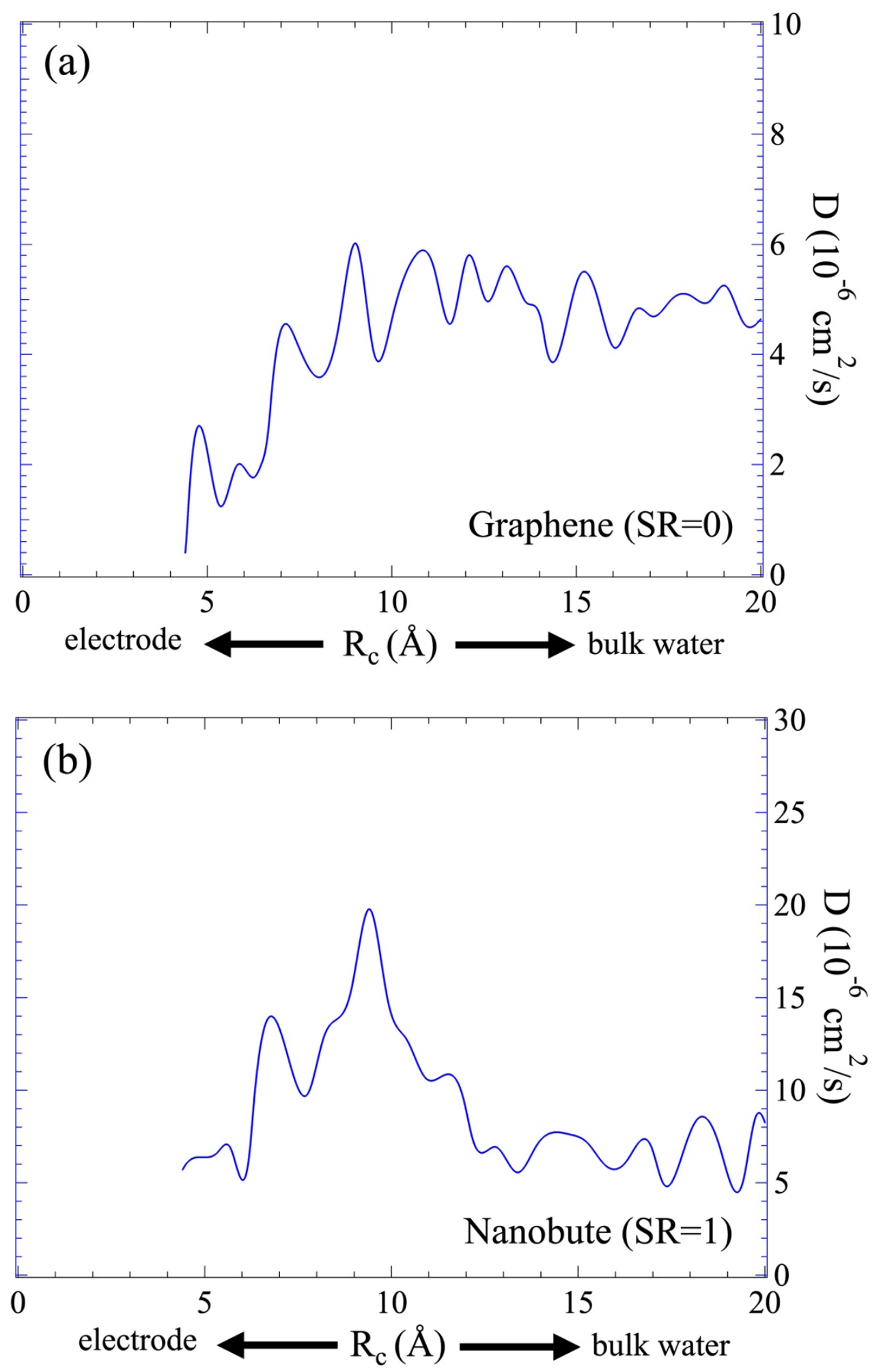

Figure 4. Calculated diffusion coefficient, D, as a function of the dopamine-electrode distance, $R_{c}$, for graphene and nanotube electrodes, respectively. 


\section{References:}

1. Bernheimer, H.; Birkmayer, W.; Hornykiewicz, O.; Jellinger, K.; Seitelberger, F., Brain dopamine and the syndromes of Parkinson and Huntington Clinical, morphological and neurochemical correlations. Journal of the neurological sciences 1973, 20, (4), 415-455.

2. Morissette, M.; Di Paolo, T., Sex and estrous cycle variations of rat striatal dopamine uptake sites. Neuroendocrinology 1993, 58, (1), 16-22.

3. Calipari, E. S.; Juarez, B.; Morel, C.; Walker, D. M.; Cahill, M. E.; Ribeiro, E.; RomanOrtiz, C.; Ramakrishnan, C.; Deisseroth, K.; Han, M.-H., Dopaminergic dynamics underlying sexspecific cocaine reward. Nature communications 2017, 8 .

4. Dayan, P., Dopamine, reinforcement learning, and addiction. Pharmacopsychiatry 2009, 42, (S 01), S56-S65.

5. Bello, E. P.; Mateo, Y.; Gelman, D. M.; Noaín, D.; Shin, J. H.; Low, M. J.; Alvarez, V. A.; Lovinger, D. M.; Rubinstein, M., Cocaine supersensitivity and enhanced motivation for reward in mice lacking dopamine D2 autoreceptors. Nature neuroscience 2011, 14, (8), 1033-1038.

6. Venton, B. J.; Robinson, T. E.; Kennedy, R. T.; Maren, S., Dynamic amino acid increases in the basolateral amygdala during acquisition and expression of conditioned fear. European Journal of Neuroscience 2006, 23, (12), 3391-3398.

7. Carboni, E.; Imperato, A.; Perezzani, L.; Di Chiara, G., Amphetamine, cocaine, phencyclidine and nomifensine increase extracellular dopamine concentrations preferentially in the nucleus accumbens of freely moving rats. Neuroscience 1989, 28, (3), 653-61.

8. Giros, B.; Jaber, M.; Jones, S. R.; Wightman, R. M.; Caron, M. G., Hyperlocomotion and indifference to cocaine and amphetamine in mice lacking the dopamine transporter. Nature 1996, $379,(6566), 606-612$.

9. Liang, B. T.; Jacobson, K. A., A physiological role of the adenosine A3 receptor: sustained cardioprotection. Proceedings of the National Academy of Sciences 1998, 95, (12), 6995-6999.

10. Ganesana, M.; Venton, B. J., Early changes in transient adenosine during cerebral ischemia and reperfusion injury. PloS one 2018, 13, (5), e0196932.

11. Zestos, A. G.; Luna-Munguia, H.; Stacey, W. C.; Kennedy, R. T., Use and Future Prospects of in Vivo Microdialysis for Epilepsy Studies. ACS chemical neuroscience 2018.

12. Crick, E. W.; Osorio, I.; Bhavaraju, N. C.; Linz, T. H.; Lunte, C. E., An investigation into the pharmacokinetics of 3-mercaptopropionic acid and development of a steady-state chemical seizure model using in vivo microdialysis and electrophysiological monitoring. Epilepsy research 2007, 74, (2-3), 116-125.

13. Song, P.; Mabrouk, O. S.; Hershey, N. D.; Kennedy, R. T., In vivo neurochemical monitoring using benzoyl chloride derivatization and liquid chromatography-mass spectrometry. Analytical chemistry 2011, 84, (1), 412-419.

14. Donzanti, B. A.; Yamamoto, B. K., An improved and rapid HPLC-EC method for the isocratic separation of amino acid neurotransmitters from brain tissue and microdialysis perfusates. Life sciences 1988, 43, (11), 913-922.

15. Yokoi, F.; Gründer, G.; Biziere, K.; Stephane, M.; Dogan, A. S.; Dannals, R. F.; Ravert, H.; Suri, A.; Bramer, S.; Wong, D. F., Dopamine D2 and D3 receptor occupancy in normal humans treated with the antipsychotic drug aripiprazole (OPC 14597): a study using positron emission tomography and [11C] raclopride. Neuropsychopharmacology 2002, 27, (2), 248-259.

16. Xiao, N.; Venton, B. J., Rapid, sensitive detection of neurotransmitters at microelectrodes modified with self-assembled SWCNT forests. Analytical chemistry 2012, 84, (18), 7816-7822. 
17. Jacobs, C. B.; Ivanov, I. N.; Nguyen, M. D.; Zestos, A. G.; Venton, B. J., High temporal resolution measurements of dopamine with carbon nanotube yarn microelectrodes. Analytical chemistry 2014, 86, (12), 5721-5727.

18. Zestos, A. G.; Jacobs, C. B.; Trikantzopoulos, E.; Ross, A. E.; Venton, B. J., Polyethylenimine Carbon Nanotube Fiber Electrodes for Enhanced Detection of Neurotransmitters. Analytical chemistry 2014, 86, (17), 8568-8575.

19. Mendoza, A.; Asrat, T.; Liu, F.; Wonnenberg, P.; Zestos, A. G., Carbon Nanotube Yarn Microelectrodes Promote High Temporal Measurements of Serotonin Using Fast Scan Cyclic Voltammetry. Sensors 2020, 20, (4), 1173.

20. Golze, D.; Iannuzzi, M.; Nguyen, M.-T.; Passerone, D.; Hutter, J., Simulation of Adsorption Processes at Metallic Interfaces: An Image Charge Augmented QM/MM Approach. Journal of Chemical Theory and Computation 2013, 9, (11), 5086-5097.

21. Warshel, A.; Levitt, M., Theoretical studies of enzymic reactions: Dielectric, electrostatic and steric stabilization of the carbonium ion in the reaction of lysozyme. Journal of Molecular Biology 1976, 103, (2), 227-249.

22. Hohenberg, P.; Kohn, W., Inhomogeneous Electron Gas. Physical Review 1964, 136, (3B), B864-B871.

23. Perdew, J. P.; Ernzerhof, M.; Burke, K., Rationale for mixing exact exchange with density functional approximations. The Journal of Chemical Physics 1996, 105, (22), 9982-9985.

24. Woon, D. E.; Dunning, T. H., Gaussian basis sets for use in correlated molecular calculations. IV. Calculation of static electrical response properties. The Journal of Chemical Physics 1994, 100, (4), 2975-2988.

25. Siepmann, J. I.; Sprik, M., Influence of surface topology and electrostatic potential on water/electrode systems. The Journal of Chemical Physics 1995, 102, (1), 511-524.

26. VandeVondele, J.; Krack, M.; Mohamed, F.; Parrinello, M.; Chassaing, T.; Hutter, J., Quickstep: Fast and accurate density functional calculations using a mixed Gaussian and plane waves approach. Computer Physics Communications 2005, 167, (2), 103-128.

27. Kumar, S.; Rosenberg, J. M.; Bouzida, D.; Swendsen, R. H.; Kollman, P. A., THE weighted histogram analysis method for free-energy calculations on biomolecules. I. The method. Journal of Computational Chemistry 1992, 13, (8), 1011-1021.

28. Hansen, N.; van Gunsteren, W. F., Practical Aspects of Free-Energy Calculations: A Review. Journal of Chemical Theory and Computation 2014, 10, (7), 2632-2647.

29. Ferrenberg, A. M.; Swendsen, R. H., Optimized Monte Carlo data analysis. Physical Review Letters 1989, 63, (12), 1195-1198.

30. Trouillon, R.; Lin, Y.; Mellander, L. J.; Keighron, J. D.; Ewing, A. G., Evaluating the Diffusion Coefficient of Dopamine at the Cell Surface During Amperometric Detection: Disk vs Ring Microelectrodes. Analytical Chemistry 2013, 85, (13), 6421-6428.

31. Lin, C.; Chen, L.; Tanner, E. E. L.; Compton, R. G., Electroanalytical study of dopamine oxidation on carbon electrodes: from the macro- to the micro-scale. Physical Chemistry Chemical Physics 2018, 20, (1), 148-157.

32. Chen, H., Functional Mode Electron-Transfer Theory. The Journal of Physical Chemistry $B$ 2014, 118, (27), 7586-7593.

33. Elenewski, J. E.; Cai, J. Y.; Jiang, W.; Chen, H., Functional Mode Hot Electron Transfer Theory. The Journal of Physical Chemistry C 2016, 120, (37), 20579-20587.

34. Levitt, D. G., General continuum theory for multiion channel. I. Theory. Biophysical Journal 1991, 59, (2), 271-277. 
35. Woolf, T. B.; Roux, B., Conformational Flexibility of o-Phosphorylcholine and oPhosphorylethanolamine: A Molecular Dynamics Study of Solvation Effects. Journal of the American Chemical Society 1994, 116, (13), 5916-5926.

36. Chen, H.; Wu, Y.; Voth, G. A., Proton Transport Behavior through the Influenza A M2 Channel: Insights from Molecular Simulation. Biophysical Journal 2007, 93, (10), 3470-3479.

37. Li, H.; Chen, H.; Steinbronn, C.; Wu, B.; Beitz, E.; Zeuthen, T.; Voth, G. A., Enhancement of Proton Conductance by Mutations of the Selectivity Filter of Aquaporin-1. Journal of Molecular Biology 2011, 407, (4), 607-620.

38. Chen, H.; Ilan, B.; Wu, Y.; Zhu, F.; Schulten, K.; Voth, G. A., Charge Delocalization in Proton Channels, I: The Aquaporin Channels and Proton Blockage. Biophysical Journal 2007, 92, (1), 46-60.

39. Randles, J. E. B., A cathode ray polarograph. Part II.-The current-voltage curves. Transactions of the Faraday Society 1948, 44, (0), 327-338.

40. Sevcik, A., Oscillographic polarography with periodical triangular voltage. Collect. Czech. Chem. Commun. 1948, 13, 349-377.

41. Ferreira, C.; López, J. L.; Miana, P., Two Algorithms for Computing the Randles-Sevcik Function from Electrochemistry. Journal of Mathematical Chemistry 2004, 35, (2), 131-137.

42. Trouillon, R.; Lin, Y.; Mellander, L. J.; Keighron, J. D.; Ewing, A. G., Evaluating the Diffusion Coefficient of Dopamine at the Cell Surface During Amperometric Detection: Disk vs. Ring Microelectrodes. Analytical chemistry 2013, 85, (13), 6421-6428.

43. Vatamanu, J.; Bedrov, D.; Borodin, O., On the application of constant electrode potential simulation techniques in atomistic modelling of electric double layers. Molecular Simulation 2017, 43, (10-11), 838-849. 